\title{
Anatomic Distribution of Cherry Angiomas in the General Population
}

\author{
Brigid Betz-Stablein ${ }^{\mathrm{a}, \mathrm{b}}$ Uyen Koh ${ }^{\mathrm{b}}$ Harrison A. Edwards $\mathrm{s}^{\mathrm{b}, \mathrm{c}}$ \\ Aideen McInerney-Leo ${ }^{b}$ Monika Janda ${ }^{d}$ H. Peter Soyer ${ }^{b, c}$ \\ ${ }^{a}$ Cancer and Population Studies, QIMR Berghofer Medical Research Institute, Brisbane, QLD, Australia; \\ bThe University of Queensland Diamantina Institute, The University of Queensland, Dermatology Research Centre, \\ Brisbane, QLD, Australia; 'Dermatology Department, Princess Alexandra Hospital, Brisbane, QLD, Australia; \\ ${ }^{d}$ Centre of Health Services Research, The University of Queensland, Faculty of Medicine, Brisbane, QLD, Australia
}

\section{Keywords}

Senile angiomas · Campbell de Morgan spots · Benign vascular tumours - Melanoma risk prediction · Skin cancer . Epidemiology

\begin{abstract}
Background: Cherry angiomas are common benign vascular skin lesions of unknown aetiology, found largely on the trunk. However, their exact anatomic distribution besides their truncal predisposition, and how they manifest in the general population, has not been characterised. Methods: Three-dimensional (3D) total body imaging was obtained from 163 adult participants of a general population cohort study in Brisbane, Australia. Demographic, phenotypic, and sun behaviour characteristics were collected using a standard questionnaire along with history of melanoma and keratinocyte cancers. Cherry angiomas were identified using an automated classification algorithm with a sensitivity of $87 \%$ and a specificity of $99 \%$, developed specifically for this study population. Results: The 3D total body images of 163 participants were analysed. Participants had a median age of 57 years and $61 \%$ were male. On average, males had more angiomas than females (median of 16 vs. 12) and the number and size of cherry angiomas increased with age. In addition to male sex and age, an increase in angiomas was associated
\end{abstract}

karger@karger.com www.karger.com/drm

Karger $\stackrel{\text { ' }}{5}$
(C) 2021 The Author(s)

Published by S. Karger AG, Basel

This is an Open Access article licensed under the Creative Commons Attribution-NonCommercial-4.0 International License (CC BY-NC) (http://www.karger.com/Services/OpenAccessLicense), applicable to the online version of the article only. Usage and distribution for commercial purposes requires written permission. with Caucasian ancestry other than British/Irish only, fair skin colour opposed to medium/olive, having green/hazel eyes compared to blue/grey, and personal history of melanoma. The most common site for cherry angiomas was the front trunk, followed by the back. Interestingly, although males had more angiomas overall, females had more angiomas on the legs. Conclusion: Describing the distribution of cherry angiomas by body site is an important step towards further understanding of the aetiology of angiomas. While personal history of melanoma is associated with an increased number of cherry angiomas, whether this association is prognostic, co-occurs with development of melanoma, or is merely fortuitous requires further investigation.

(c) 2021 The Author(s).

Published by S. Karger AG, Basel

\section{Introduction}

Cherry angiomas (also called Campbell de Morgan spots or, less elegantly, senile angiomas) are amongst the spectrum of benign lesions that adorn the cutaneous landscape. They generally present as round, bright red macules that vary in size up to $5 \mathrm{~mm}$ [1]. While cherry

This article is part of the Nevi Article Series.
Correspondence to:

Brigid Betz-Stablein, b.betzstablein@uq.edu.au Monika Janda, m.janda@uq.edu.au 
angiomas are a common vascular proliferation, their aetiology remains largely unknown. One potential aetiology is the exposure to risk factors including chemicals, sulphur gas, and infection by herpesvirus 8 and varicella zoster virus $[2,3]$. The second is that new (somatic) genetic mutations occur in the skin [4]. This has recently been supported by the identification of somatic GNAQ and GNA11 mutations in cherry angiomas [5], a genetic hotspot associated with other conditions such as uveal melanoma, blue naevi, and blue naevus-associated melanoma [5].

Cherry angiomas are common, with $54 \%$ of Australian adults ( $>20$ years) having at least one [6]. The prevalence increases from $22 \%$ in people aged $20-30$ years to $40-78 \%$ in those older than $70[6,7]$. An Australian study $(n=$ $1,457)$ showed that women were somewhat more likely to have one or more cherry angiomas than men (58 vs. $50 \%$ ). Conversely, a small Turkish study $(n=187)$ in nursing home residents reported a higher prevalence of angiomas in men than women (50 vs. 33\%) [7], and a 1947 Scottish study of 1,300 hospital patients of all ages reported no sex differences in the presence or absence of cherry angiomas [1]. "Eruptive" angiomas is a condition referring to having multiple cherry angiomas. However, the term is not well defined, with a prevalence in adults varying from 5 to $74 \%$ depending on age and what is considered "multiple," with cut-offs ranging from $>10$ to $>50$ of these lesions $[2,8,9]$. Factors associated with having $>30$ cherry angiomas include increasing age, male sex, immunosuppressive treatment, and cutaneous and extracutaneous malignancies [2].

While cherry angiomas are the most common benign vascular tumour in humans, their exact anatomic distribution besides their truncal predisposition has not been characterised. One study quantified the number of cherry angiomas on the abdominal region, and on this specific location it was shown that the frequency and size of cherry angiomas increases with age [1]. Hence, in our study, we used machine learning and three-dimensional (3D) total body imaging to describe the frequency and anatomic distribution of cherry angiomas in members of the general population recruited from the Brisbane electoral roll (a population-based registry of adults aged $\geq 18$ years) and their association with demographic, phenotypic, and sun behaviour characteristics. A better understanding of the prevalence of cherry angiomas, and how they manifest within a person and the general population, may contribute to the knowledge base of their aetiology.

\section{Subjects and Methods}

\section{Study Participants}

The "Mind your Moles" study was designed to investigate the natural history of naevi, with detailed study processes and procedures described elsewhere [10]. Figure 1 outlines the methodology $[11,12]$. Briefly, we recruited adults aged $20-69$ years living in the greater Brisbane electoral role. The inclusion criteria required participants to be able to provide informed consent, have at least one naevus, Fitzpatrick skin type I-IV, and agreed to participate in a 3-year longitudinal study with 6-monthly clinic visits for imaging of their total body surface. There were no additional exclusion criteria [10].

\section{Data Collection}

At the baseline clinic visit for the "Mind your Moles" study, a research assistant administered a standard questionnaire to obtain information about ancestry, occupation, sun behaviour, and skin cancer history. Participants' height, weight, as well as hair, eye, and skin colour were also recorded. At follow-up visits, new diagnoses of skin cancer were obtained.

For this study, imaging data were obtained from the 18-month time point of the general population cohort study. The 18-month clinic time point was chosen as this was the first time point where all participants were scanned with the newest 3D total body scanner (VECTRA WB360 Serial Number WB00009; Canfield Scientific, Parsippany, NJ, USA). This technology stitches 92 simultaneously taken photos into a 3D total body model which we refer to as a 3D avatar [13], allowing detailed assessment of the total body surface except for areas covered by hair, underwear, or soles of the feet.

\section{Preparation of Training and Test Set}

A clinician identified all cherry angiomas on 20 participants. Four of the participants were selected as they had previously been observed to have many cherry angiomas $(>120)$, and the other 16 were randomly selected from the population. To ensure an even number of men and women the sample function in $\mathrm{R}$ was used to randomly select 10 women and 6 men without replacement, as the 4 with $>120$ cherry angiomas were all male. The 20 participants were spilt 60:40 into a training and a test set. The 4 participants included for their large number of angiomas were split 3:1 (training:test). Three females and 2 males were randomly selected from the remaining 16 participants to complete the test set. The characteristics of the training and the test set are presented in online supplementary Table 1 (for all online suppl. material, see www.karger.com/doi/10.1159/000517172).

\section{Classification Algorithm}

The $3 \mathrm{D}$ images were divided into image tiles (approximately 32 $\times 32$ to $150 \times 150$ pixels each). The training set consisted of 882 cherry angiomas, 7,167 other skin lesions, and 161,013 non-lesions. The test set consisted of 334 cherry angiomas, 2,199 other skin lesions, and 54,326 non-lesion images. Fifty-three variables such as the presence or absence of a lesion, colour, contrast between the lesion and perilesional skin, and lesion diameter were extracted from images and saved in JavaScript Object Notation (JSON) format. These variables were entered as the input for the classification tree which was fitted in R using the package rpart [11] and caret [14]. The extreme gradient boosting method (xbgTree) 
Fig. 1. The study population and methodology. 3D, three-dimensional.
Data: 3D total body images obtained from 163 participants who attended their 18month* visit from the longitudinal "Mind your Moles" study primarily designed to study the natural history of naevi [10]

Image labelling: 1,226 cherry angiomas identified by clinician observation on 20 of the 3D total body images (including 16 randomly selected; 4 with many angiomas)
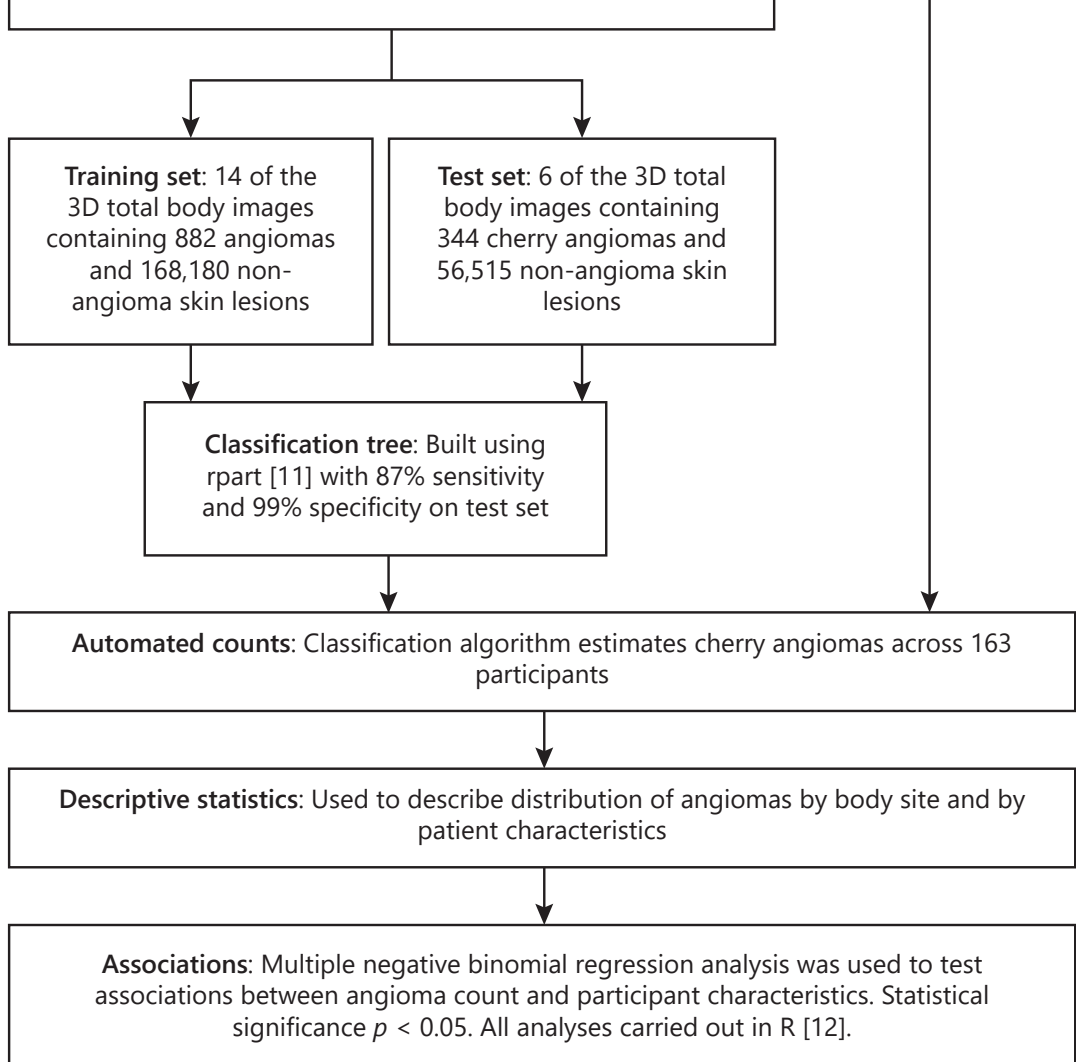

*The 18-month clinic time point was chosen as this was the first time point where all participants were scanned with the newest 3D total body scanner (VECTRA WB360 Serial Number WB00009, Canfield Scientific, Parsippany, NJ, USA). was used to find the optimal classification algorithm. The sensitivity of the algorithm on the test set was $87 \%$ with a specificity of $99 \%$. Kappa was 0.88 , which suggests almost perfect agreement between the clinician and the automated classification. No notable differences were observed in the algorithm's performance across different participant characteristics (data not show).

\section{Statistical Analysis}

Descriptive statistics were used to summarise participant characteristics, with categorical variables summarised using frequencies and percentages, and cherry angioma counts summarised using medians and interquartile ranges. Negative binomial regression was used to investigate characteristics associated with cherry angioma count. Variables with univariate $p$ values $<0.2$ were included in a multivariate model with backwards selection used to refine the model. All analyses were carried out in R [12].

\section{Results}

3D total body images for 163 general population adults in Brisbane, Australia were included in the analysis [15]. Sixty-one per cent were male and the median age was 57 years (range, 25-72 years). Most participants (65\%) re- 
Table 1. Median number of cherry angiomas by participant characteristics

\begin{tabular}{|c|c|c|}
\hline Characteristic & $\begin{array}{l}n(\%) \\
(N=163)\end{array}$ & $\begin{array}{l}\text { Angiomas, } \\
\text { median (range) }\end{array}$ \\
\hline \multicolumn{3}{|l|}{ Sex } \\
\hline Female & $63(39 \%)$ & $12(0-85)$ \\
\hline Male & $100(61 \%)$ & $16(0-297)$ \\
\hline \multicolumn{3}{|l|}{ Age } \\
\hline$<50$ years & $44(27 \%)$ & $10(0-92)$ \\
\hline $50-60$ years & $54(33 \%)$ & $14(2-151)$ \\
\hline$>60$ years & $65(40 \%)$ & $19(1-297)$ \\
\hline \multicolumn{3}{|l|}{ Ancestry } \\
\hline British/Irish only & $107(66 \%)$ & $12(0-297)$ \\
\hline Other & $56(34 \%)$ & $20(1-164)$ \\
\hline \multicolumn{3}{|l|}{ Innate skin colour } \\
\hline Fair & $126(77 \%)$ & $16(0-297)$ \\
\hline Medium/olive & $36(22 \%)$ & $13(0-164)$ \\
\hline \multicolumn{3}{|l|}{ Eye colour } \\
\hline Blue or grey & $84(52 \%)$ & $13(0-224)$ \\
\hline Green or hazel & $52(32 \%)$ & $25(0-297)$ \\
\hline Brown & $26(16 \%)$ & $14(0-101)$ \\
\hline \multicolumn{3}{|c|}{ Skin reaction to acute sun } \\
\hline Only tan & $15(9 \%)$ & $16(2-43)$ \\
\hline Burn then tan & $106(65 \%)$ & $14(0-297)$ \\
\hline Always burn & $42(26 \%)$ & $18(0-224)$ \\
\hline \multicolumn{3}{|c|}{ Number of moles $\geq 2 \mathrm{~mm}$} \\
\hline Few $(0-20)$ & $104(64 \%)$ & $14(0-224)$ \\
\hline Some (21-50) & $36(22 \%)$ & $16(0-297)$ \\
\hline Many $(>50)$ & $19(12 \%)$ & $22(2-114)$ \\
\hline \multicolumn{3}{|l|}{ Freckling score } \\
\hline None/mild & $92(56 \%)$ & $16(0-164)$ \\
\hline Moderate/severe & $71(44 \%)$ & $13(0-297)$ \\
\hline \multicolumn{3}{|c|}{ Number of burns before age 20} \\
\hline$\leq 10$ & $63(39 \%)$ & $13(1-297)$ \\
\hline$>10$ & $100(61 \%)$ & $16(0-224)$ \\
\hline \multicolumn{3}{|c|}{ Family history of melanoma } \\
\hline No & $121(74 \%)$ & $16(0-224)$ \\
\hline Yes & $42(26 \%)$ & $12(0-297)$ \\
\hline \multicolumn{3}{|c|}{ Personal history of melanoma } \\
\hline No & $152(93 \%)$ & $21(0-160)$ \\
\hline Yes & $11(7 \%)$ & $51(5-297)$ \\
\hline \multicolumn{3}{|c|}{ Keratinocyte skin cancer } \\
\hline No & $114(70 \%)$ & $26(0-164)$ \\
\hline Yes & $49(30 \%)$ & $32(1-297)$ \\
\hline
\end{tabular}

ported British/Irish ancestry. Additional participant characteristics can be found in Table 1. Cherry angiomas were more numerous in men than women, with a median of 16 (range, 0-297), and 12 (range, 0-85), respectively (Fig. 2). Sixty-six per cent of our population had $>10$ cherry angiomas $(>1 \mathrm{~mm})$ consistent with one definition of "eruptive" cherry angiomas [8]. However, using 30 cherry angiomas [2] or 50 cherry angiomas [9] as the thresh- old would result in $27 \%$ or $16 \%$ of the population, respectively, being diagnosed (Table 2). If only cherry angiomas $>2 \mathrm{~mm}$ are considered, the proportions decrease further to 24,6 , and $2 \%$, respectively, for the cut-offs 10,30 , and 50.

The most common site for cherry angiomas was the front torso area with a median of 8 (range, $0-123$ ) for men and 5 (range, $0-40$ ) for women (Fig. 3; Table 3). This was followed by the back with a median of 5 (range, $0-112$ ), and 4 (range, $0-30$ ) for men and women, respectively. Similar numbers of cherry angiomas were seen on the arms (1 [range, 0-34]) and legs (1 [range, 0-29]), with the lowest number of angiomas found on the head and neck (0 [range, 0-8]) (Table 3; Fig. 3). Counts from either the front torso, back torso, or arms only showed high correlation $(0.96,0.96$, and 0.87 , respectively) with total body angioma counts (Table 4). While men had a higher number of angiomas overall and on most individual body sites, women appeared to have a slightly higher median number of cherry angiomas on the legs (Table 3).

A multivariate negative binomial model explored associations between participant characteristics and cherry angioma count. Being male, increasing in age, having fair innate skin colour, being of Caucasian ancestry other than British/Irish, and having green/hazel eye colour as opposed to blue as well as a personal history of melanoma were all associated with an increase in the number of cherry angiomas (Table 5). Specifically, every 1 -year increase in age is expected to result in a $4 \%$ increase in the number of cherry angiomas; for an increase in 10 years this corresponds to an increase by $48 \%\left(1.04^{10}\right)$. Compared to those with British/Irish ancestry only, those with a combination of other ancestries were expected to have 2.2 times the number of cherry angiomas. However, those with medium/olive innate skin tone were expected to have a $35 \%$ decrease in the number of cherry angiomas compared to those with fair innate skin colour. People with green/hazel eyes had $87 \%$ more cherry angiomas compared to those with blue/grey eyes. Those who had a personal history of melanoma showed the largest association to number of cherry angioma and were expected to have 3.15 times as many cherry angiomas as those who did not have a personal history of melanoma. No relationship was observed between the number of cherry angiomas and $>20$ self-reported sunburns before the age of 20 , skin reaction to first annual exposure to acute sun, freckling, number of naevi, past keratinocyte cancer, or self-reported family history of melanoma. 


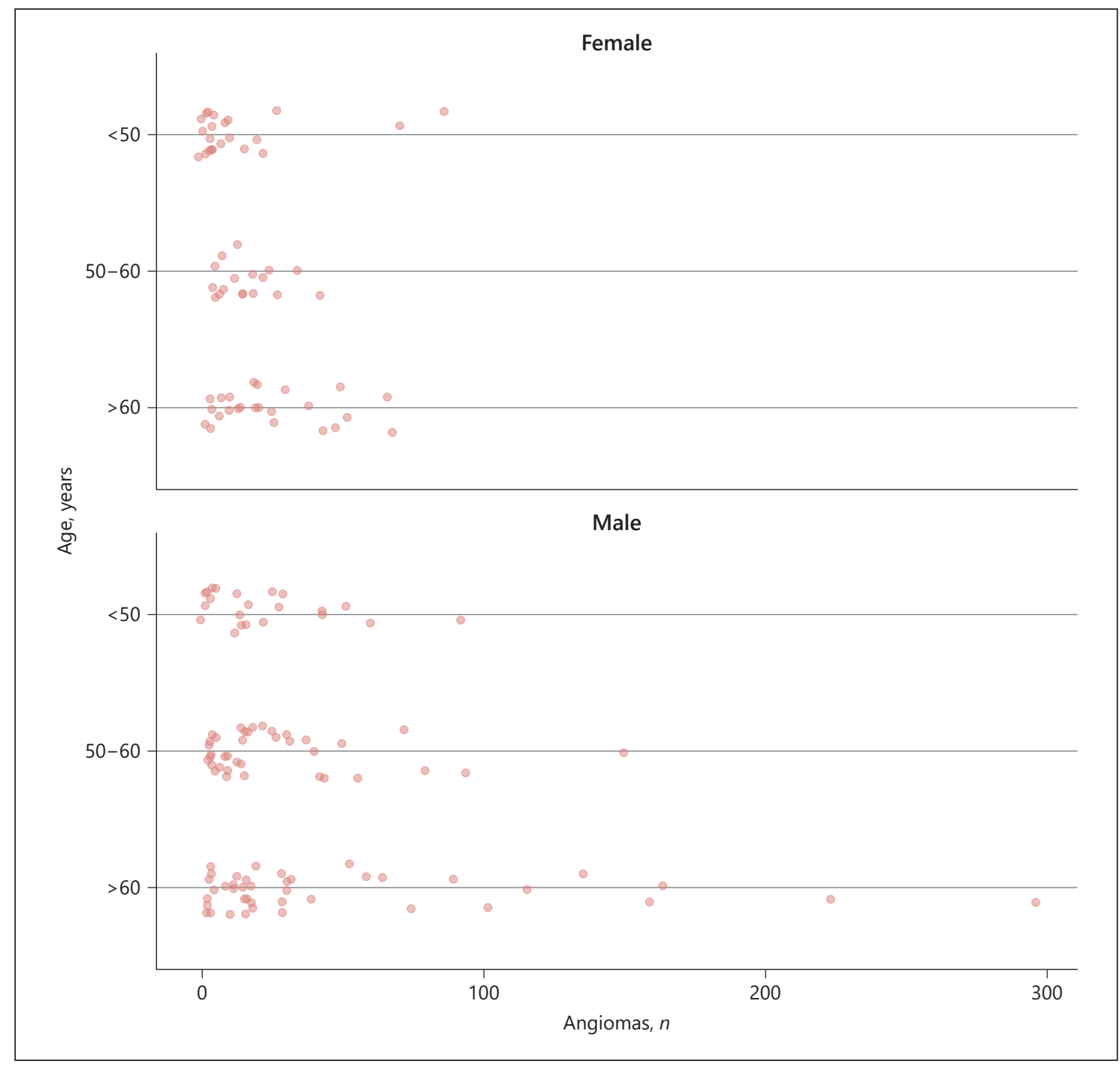

Fig. 2. Number of cherry angiomas by sex and age. Each dot represents counts for one person.

\section{Discussion}

We present a robust methodology to automatically assess the size and body site distribution of cherry angiomas, and applied this methodology to 163 randomly selected participants from the Brisbane, Australia adult general population [15]. This was largely assisted by the exact anatomic documentation provided by the $3 \mathrm{D}$ total body photography and the ability to apply automated algorithms directly to these images. Previous counts of cherry angiomas in the literature have only been provided for the abdominal area [3]. The majority of studies simply describe the absence or presence of one or more cherry angiomas, or the presence/absence of "eruptive" angiomas (with arbitrary cut-offs of 10 [8], 30 [2], or 50 [9]). This study shows that the proportion of people classified as having "eruptive" angiomas can vary greatly depending on the criteria selected. In our participants, we found that the majority (66\%) of subjects had $>10$ angiomas $>1$ $\mathrm{mm}$, but this decreased to $24 \%$ if only angiomas $>2 \mathrm{~mm}$ were considered. Our method overcomes the necessity to use arbitrary cut-offs by allowing exact counts, their anatomic location, and size to be automatically obtained. Moreover, the exact coordinates map the cherry angiomas directly to the 3D total body image, allowing the anatomic distribution to be described in unprecedented de- 
Table 2. Frequency and percentage of participants classified as having "eruptive" cherry angiomas, with differing cut-offs for both size $(>1 \mathrm{~mm}$ or $>2 \mathrm{~mm})$ and frequency $(\geq 10, \geq 30$, or $\geq 50)$

\begin{tabular}{|c|c|c|c|c|c|c|}
\hline "Eruptive" cut-off & \multicolumn{6}{|c|}{ Prevalence of "eruptive" cherry angiomas (>1 mm), $n(\%)$} \\
\hline Female & $27(42 \%)$ & $36(57 \%)$ & $51(81 \%)$ & $12(19 \%)$ & $58(92 \%)$ & $5(8 \%)$ \\
\hline Male & $29(29 \%)$ & $71(71 \%)$ & $68(68 \%)$ & $32(32 \%)$ & $79(79 \%)$ & $21(21 \%)$ \\
\hline $50-60$ years & $19(35 \%)$ & $35(65 \%)$ & $40(74 \%)$ & $14(26 \%)$ & $48(89 \%)$ & $6(11 \%)$ \\
\hline \multirow[t]{2}{*}{$>60$ years } & $16(25 \%)$ & $49(75 \%)$ & $42(65 \%)$ & $23(36 \%)$ & $50(77 \%)$ & $15(23 \%)$ \\
\hline & \multicolumn{6}{|c|}{ Prevalence of "eruptive" cherry angiomas (>2 mm), $n(\%)$} \\
\hline "Eruptive" cut-off & $<10$ & $\geq 10$ & $<30$ & $\geq 30$ & $<50$ & $\geq 50$ \\
\hline Male & $72(72 \%)$ & $28(28 \%)$ & $90(90 \%)$ & $10(10 \%)$ & $96(96 \%)$ & $4(4 \%)$ \\
\hline \multicolumn{7}{|l|}{ Age } \\
\hline$<50$ years & $37(84 \%)$ & $7(16 \%)$ & $43(98 \%)$ & $1(2 \%)$ & $44(100 \%)$ & $0(0 \%)$ \\
\hline $50-60$ years & $43(80 \%)$ & $11(20 \%)$ & $53(98 \%)$ & $1(1 \%)$ & $54(100 \%)$ & $0(0 \%)$ \\
\hline$>60$ years & $44(68 \%)$ & $21(32 \%)$ & $57(88 \%)$ & $8(12 \%)$ & $61(94 \%)$ & $4(6 \%)$ \\
\hline
\end{tabular}

Table 3. Number of cherry angiomas by body site, sex; and age

\begin{tabular}{|c|c|c|c|c|c|c|c|c|}
\hline & \multicolumn{8}{|c|}{ Median number of cherry angiomas (range) } \\
\hline & all & $<50$ years & $50-60$ years & $>60$ years & all & $<50$ years & $50-60$ years & $>60$ years \\
\hline Number & 100 & & & & 63 & & & \\
\hline Whole body & $16(0-297)$ & $14(0-92)$ & $15(2-151)$ & $18(1-297)$ & $12(0-85)$ & $4(0-85)$ & $13(4-42)$ & $20(2-67)$ \\
\hline Head and neck & $0(0-8)$ & $0(0-4)$ & $0(0-5)$ & $1(0-8)$ & $0(0-3)$ & $0(0-3)$ & $0(0-2)$ & $0(0-3)$ \\
\hline Front torso & $8(0-123)$ & $6(0-38)$ & $8(0-72)$ & $12(0-123)$ & $5(0-40)$ & $2(0-40)$ & $6(0-13)$ & $8(0-24)$ \\
\hline Back torso & $5(0-112)$ & $4(0-39)$ & $5(0-50)$ & $8(0-112)$ & $4(0-30)$ & $2(0-30)$ & $5(0-22)$ & $5(0-21)$ \\
\hline Legs & $0(2-29)$ & $0(0-4)$ & $0(0-21)$ & $1(0-29)$ & $2(0-22)$ & $1(0-22)$ & $2(1-14)$ & $2(0-22)$ \\
\hline
\end{tabular}

Overall median number of cherry angiomas: 15 (range, 0-297).

tail. Similar to previous reports, we show that the highest number of angiomas are located on the front torso and that both the number and size of cherry angiomas increase with age [1]. Cherry angiomas are present in higher frequencies in men across all body sites, with the exception of the legs, where females have slightly more. A limitation to describing the full anatomic distribution is the fact that participants wore underwear during imaging, which prevents assessments of these regions of the body,
Table 4. Correlation between total angioma counts and body site counts

\begin{tabular}{ll}
\hline Body site & Correlation $(95 \% \mathrm{CI})$ \\
\hline Head and neck & $0.47(0.33-0.58)$ \\
Arms & $0.87(0.82-0.90)$ \\
Front & $0.96(0.94-0.97)$ \\
Back & $0.96(0.95-0.97)$ \\
Legs & $0.65(0.56-0.74)$ \\
\hline
\end{tabular}




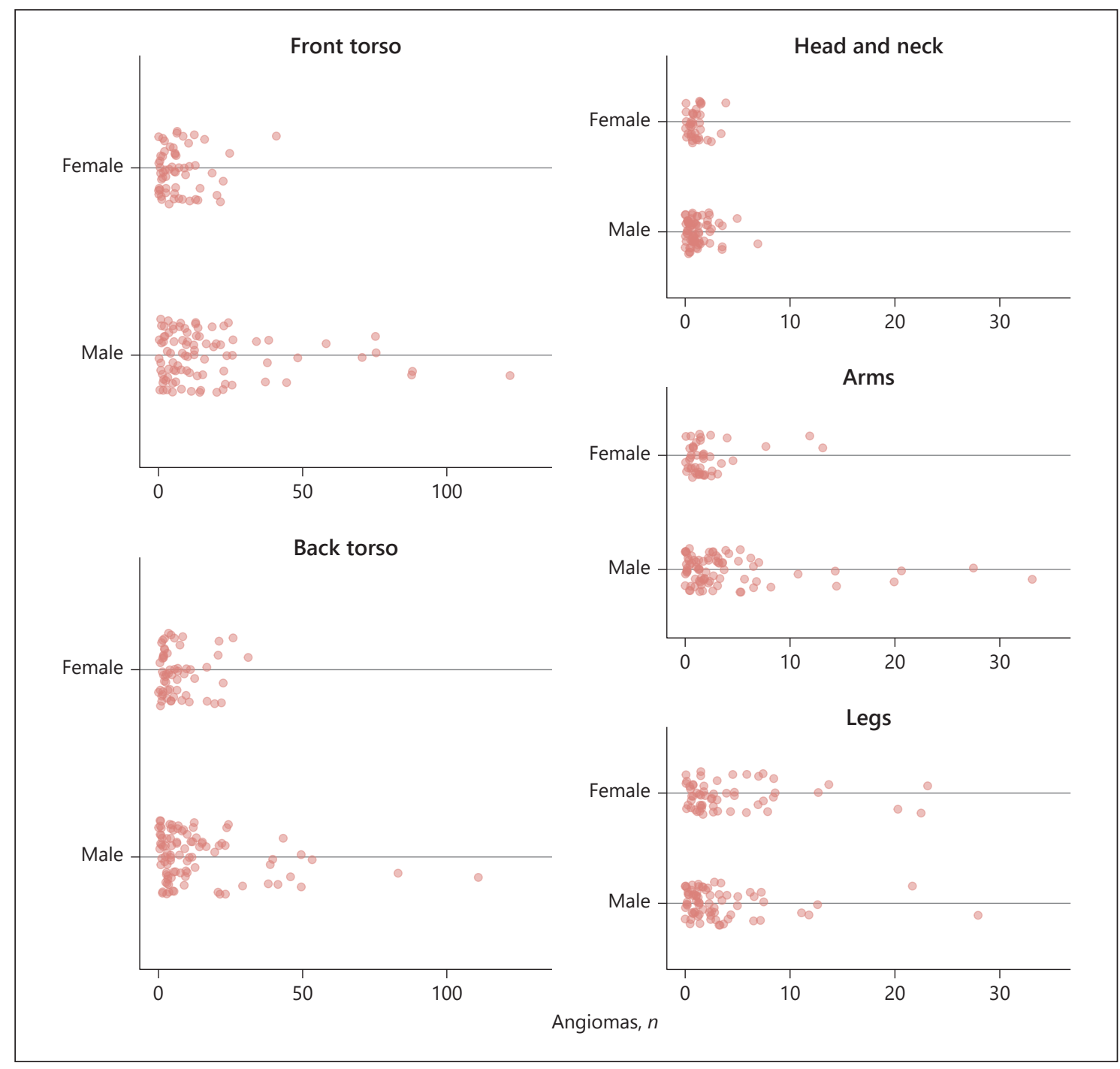

Fig. 3. Distribution of cherry angiomas by sex and body site. Each dot represents counts for one person.

such as the breast in females, where an association with breast cancer has been described [3].

In our study of 163 participants, a higher number of cherry angiomas was significantly associated with increasing age, male sex, being of heritage other than British/Irish only, fair skin colour (opposed to medium/olive), having green/hazel eyes (compared to blue/grey), and a personal history of melanoma. Previous studies showed inconsistent results regarding the association between male sex and the presence or absence of cherry angiomas $[1,6]$; however, having multiple ("eruptive") cherry angiomas is more prevalent in men $[7,8]$. Like others, we observed increases in size and number of cherry angiomas with age $[1,4]$. While few studies have investigated race or ancestry in relation to the number of cherry angiomas, a higher number of West Scottish hospital patients recorded presence (vs. absence) of cherry angiomas compared to British Royal Airforce personnel, after accounting for age. Unlike other studies, we did not find an association between keratinocyte cancers and increasing number of cherry angiomas $[2,8]$. This could be due to the difference in the categorisation of the number of angiomas or a lack of statistical power. A number of $>50$ cherry angiomas has been suggested as a potential marker of actinic skin damage [9]; however, we did not see an association with $>20$ self-reported sunburns before the age of 20 or skin reaction to first annual exposure to acute sun in our general population study. The limitations of this analysis include the rel- 
Table 5. Multivariate negative binomial regression

\begin{tabular}{lll}
\hline Characteristic & $\begin{array}{l}\text { Prevalence rate } \\
\text { ratio (95\% CI) }\end{array}$ & $\begin{array}{l}\text { Adjusted* prevalence } \\
\text { rate ratio (95\% CI) }\end{array}$ \\
\hline $\begin{array}{l}\text { Age, continuous years } \\
\text { Sex }\end{array}$ & $1.04(1.02-1.06)$ & $1.04(1.02-1.05)$ \\
$\quad \begin{array}{l}\text { Female } \\
\text { Male }\end{array}$ & 1.00 & 1.00 \\
Ancestry & $1.88(1.32-2.67)$ & $1.38(1.01-1.84)$ \\
$\quad \begin{array}{l}\text { British/Irish only } \\
\text { Caucasian other }\end{array}$ & 1.00 & 1.00 \\
$\begin{array}{l}\text { Innate skin colour } \\
\quad \text { Fair }\end{array}$ & $1.47(1.03-2.13)$ & $2.19(1.60-3.04)$ \\
$\quad \begin{array}{l}\text { Medium/olive } \\
\text { Eye colour }\end{array}$ & 1.00 & 1.00 \\
$\quad \begin{array}{l}\text { Blue or grey } \\
\text { Green or hazel }\end{array}$ & $0.73(0.49-1.13)$ & $0.65(0.46-0.95)$ \\
$\quad$ Brown & 1.00 & 1.00 \\
Personal history of melanoma & $1.52(1.03-2.25)$ & $1.87(1.34-2.62)$ \\
$\quad$ No & $0.88(0.54-1.48)$ & $1.03(0.68-1.61)$ \\
$\quad$ Yes & 1.00 & 1.00 \\
\hline
\end{tabular}

* Prevalence rate ratios are adjusted for all other characteristics in the table. atively small sample size and potential recall bias in factors such as history of sunburns. In addition, as this study was designed specifically to investigate naevi, information was not collected on potential risk factors for cherry angiomas such as immunosuppression.

Immunosuppression, herpesvirus 8 , graft versus host disease, and exposure to chemicals [16-18] have been associated with development of multiple ("eruptive") cherry angiomas. More recently, an association between melanoma and presence of $>10$ cherry angiomas was reported in a study of 1,693 Italian patients attending outpatient dermatology clinics [8]. A follow-up study by the same authors confirmed this association and showed it to be of similar size (OR 2.26, 95\% CI 1.74-2.93) to the association between melanoma and $>2$ atypical naevi (OR 2.07, 95\% CI 1.58-2.71) [19]. Both studies were retrospective, and thus it is unknown whether the angiomas predated the melanoma or vice versa. Of note, a recent prospective study in Spain showed that having $>50$ cherry angiomas was predictive of developing a second melanoma [9]. Additionally, $70 \%$ of participants in a case series of 50 patients with unilateral breast cancer had a higher number of cherry angiomas on the breast affected with cancer than the unaffected breast prior to surgical staging [3].

$3 \mathrm{D}$ total body imaging provides the opportunity to examine the total skin surface and describe the composition of the lesion ecosystem in unprecedented detail. Describing the distribution of cherry angiomas by body site is an important step towards further understanding their natural history. While a few studies suggest that an association between cutaneous malignancies and multiple cherry angiomas is more than incidental, further studies are needed to test whether these associations are causal and prognostic, and whether development of cherry angiomas co-occurs with development of melanoma or is merely an epiphenomenon.

\section{Key Message}

We describe the anatomic distribution of cherry angiomas based on three-dimensional total body imaging and their associated characteristics.

\section{Acknowledgements}

We would like to acknowledge the clinic staff and clinicians for their assistance in collecting the data and thank our study participants for giving up their time for our research.

\section{Statement of Ethics}

Ethics approval was obtained from the Human Research Ethics Committees of Metro South Health (HREC/16/QPAH/125), the University of Queensland (2016000554), the Queensland University of Technology (1600000515), and the QIMR Berghofer Medical Research Institute (P2271).Written informed consent was obtained from participants to participate in the study. 


\section{Conflict of Interest Statement}

H.P. Soyer is shareholder of e-derm consult $\mathrm{GmbH}$ and MoleMap by Dermatologists Pty Ltd. He provides teledermatological reports regularly for both companies. He also consults for Canfield Scientific, Inc. and is an adviser of First Derm ${ }^{\mathrm{TM}}$ and Revenio Research Oy.

\section{Funding Sources}

This work was supported by the National Health and Medical Research Council - Centre of Research Excellence scheme (grant No.: APP1099021). H.P. Soyer is also funded by the Medical Re- search Future Fund - Next Generation Clinical Researcher's Program Practitioner Fellowship (APP1137127). M. Janda is funded by NHMRC TRIP Fellowship (APP1151021). A. McInerney-Leo is funded by NHMRC Fellowship (APP1158111).

\section{Author Contributions}

All authors made contributions to the development of this study and all were involved in drafting and critically revising the manuscript.

\section{References}

1 Murison AR, Sutherland JW, Williamson AM. De Morgan's spots. Br Med J. 1947; 1(4505):634-6.

2 Borghi A, Minghetti S, Battaglia Y, Corazza M. Predisposing factors for eruptive cherry angiomas: new insights from an observational study. Int J Dermatol. 2016;55(11):e598600 .

3 Guastafierro A, Verdura V, Di Pace B, Faenza $M$, Rubino C. The influence of breast cancer on the distribution of cherry angiomas on the anterior thoracic wall: a case series study. Dermatology. 2019;235(1):65-70.

4 Gao XH, Wang LL, Zhang L, Hong YX, Wei $\mathrm{H}$, Chen HD. Familial nevus flammeus associated with early onset cherry angiomas. Int J Dermatol. 2008;47(12):1284-6.

5 Klebanov N, Lin WM, Artomov M, Shaughnessy M, Njauw CN, Bloom R, et al. Use of targeted next-generation sequencing to identify activating hot spot mutations in cherry angiomas. JAMA Dermatol. 2019;155(2): 211-5.

6 Plunkett A, Merlin K, Gill D, Zuo Y, Jolley D, Marks R. The frequency of common nonmalignant skin conditions in adults in central Victoria, Australia. Int J Dermatol. 1999, 38(12):901-8
7 Özer İ, Temiz SA, Ataseven A. Prevalence of dermatological diseases in nursing home residents and their correlation with gender and comorbid diseases. Turk J Dermatol. 2019; 13(1):8-12.

8 Corazza M, Dika E, Maietti E, Musmeci D, Patrizi A, Borghi A. Eruptive cherry angiomas and skin melanoma: a fortuitous association? Melanoma Res. 2019;29(3):313-7.

9 Pastor-Tomás N, Martínez-Franco A, Bañuls J, Peñalver JC, Traves V, García-Casado Z, et al. Risk factors for the development of a second melanoma in patients with cutaneous melanoma. J Eur Acad Dermatol Venereol. 2020;34(10):2295-302.

10 Koh U, Janda M, Aitken JF, et al. "Mind your Moles" study: protocol of a prospective cohort study of melanocytic naevi. BMJ Open. 2018;8(9):e025857.

11 Therneau T, Atkinson B. rpart: Recursive Partitioning and Regression Trees. R package version 4.1-15. https://CRAN.R-project.org/ package $=$ rpart.

12 R Foundation for Statistical Computing. R: a language and environment for statistical computing. Vienna: R Foundation for Statistical Computing. https://www.R-project.org.
13 Rayner JE, Laino AM, Nufer KL, Adams L, Raphael AP, Menzies SW, et al. Clinical perspective of 3D total body photography for early detection and screening of melanoma. Front Med (Lausanne). 2018;5:152.

14 Max K. caret: Classification and Regression Training. R package version 6.0-86. https:// CRAN.R-project.org $/$ package $=$ caret.

15 Koh U, Janda M, Aitken JF, Duffy DL, Menzies S, Sturm RA, et al. "Mind your Moles" study: protocol of a prospective cohort study of melanocytic naevi. BMJ Open. 2018;8(9): e025857.

16 Raymond LW, Williford LS, Burke WA Eruptive cherry angiomas and irritant symptoms after one acute exposure to the glycol ether solvent 2-butoxyethanol. J Occup Environ Med. 1998;40(12):1059-64.

17 Cohen AD, Cagnano E, Vardy DA. Cherry angiomas associated with exposure to bromides. Dermatology. 2001;202(1):52-3.

18 Firooz A, Komeili A, Dowlati Y. Eruptive melanocytic nevi and cherry angiomas secondary to exposure to sulfur mustard gas. J Am Acad Dermatol. 1999;40(4):646-7.

19 Borghi A, Dika E, Maietti E, Scuderi V, Forconi R, Patrizi A, et al. Eruptive cherry angiomas and skin melanoma: further insights into an intriguing association. Dermatology. 2020. doi: 10.1159/000511101. Online ahead of print. 